\title{
Desarrollo del pensamiento algebraico en estudiantes de bachillerato a través de la generalización visual de sucesiones de figuras
}

\section{Development of Algebraic Thinking in High School Students through the Visual Generalization of Figural Sequences}

\author{
Jesús Valenzuela García ${ }^{1}$ \\ Victoria Eugenia Gutiérrez Marfileño²
}

\begin{abstract}
Resumen. Este artículo muestra resultados de investigación cuyo objetivo fue indagar el tipo de estrategias utilizadas en la obtención de la regla general y promover la estrategia visual para inducir un patrón en tareas de sucesiones aritméticas de figuras como vía en el desarrollo del pensamiento algebraico en 30 estudiantes de bachillerato en México. La investigación adoptó el método de Experimento de Enseñanza e implicó una fase diagnóstica y un posterior proceso iterativo de planificación, ejecución y evaluación en cuatro sesiones de intervención. A partir de la preponderancia de estrategias de naturaleza aritmética evidenciada en la fase diagnóstica, en los resultados de la tercera sesión que aquí se reporta se logró mostrar la eficacia de la estrategia visual para inducir y expresar un patrón de regularidad a partir del análisis de términos particulares de la sucesión. Los hallazgos sugieren un tipo de enseñanza que promueva la habilidad de establecer reglas generales mediante la estrategia visual.
\end{abstract}

Palabras clave: pensamiento algebraico; generalización visual; patrones; visualización; regla general.

Fecha de recepción: 16 de agosto de 2017. Fecha de aceptación: 25 de enero de 2018.

1 Departamento de Educación, Universidad Autónoma de Aguascalientes (México). jesvalgar@gmail.com

2 Departamento de Educación, Universidad Autónoma de Aguascalientes (México). vegutier@correo.uaa.mx 


\begin{abstract}
This article shows research results whose objectives were to investigate the type of strategy used in obtaining the general rule and to promote the visual strategy to induce the general rule of patterns in tasks of arithmetic sequences as a way in the development of algebraic thinking in 30 students of a high school in Mexico. The research adopted the Teaching Experiment method and involved a diagnostic phase and a subsequent iterative process of planning, executing and evaluating of four intervention sessions. From the preponderance of arithmetic strategies as a heuristic nature to establish the general rule, evidenced in the diagnostic phase, in the results of the third intervention session reported, it was possible to show the visual strategy effectiveness in the capacity of the students to induce and express the general rule from the analysis of particular terms of succession. The findings suggest a type of teaching that promotes the ability to establish general rules through visual strategy.
\end{abstract}

Keywords: algebraic thinking; visual generalization; patterns; visualization; general rule.

\title{
INTRODUCCIÓN
}

Diversas investigaciones señalan que el trabajo sobre la generalización de patrones (Cañadas, Castro \& Castro, 2008; Radford, 2010; Vergel, 2015; Callejo, García-Reche \& Fernández, 2016) puede ser un poderoso vehículo para la comprensión de relaciones entre cantidades que subyacen a la matemática funcional, contribuyendo de esta forma al establecimiento de relaciones de variación y fluidez en el manejo del lenguaje simbólico; en términos generales, del desarrollo del pensamiento algebraico (Blanton \& Kaput, 2011; Mason, Grahan \& Johnston-Wilder, 2012).

No obstante, la enseñanza tradicional del álgebra escolar, reducida a la manipulación de expresiones simbólicas y solución de problemas ficticios, no ha sido eficaz al momento de desarrollar la capacidad de abstraer e inducir relaciones generales y estructuras (Molina, 2009). Esta forma tradicional de enseñar el álgebra presupone que la habilidad de establecer relaciones matemáticas, que son el objeto y característica esencial del pensamiento algebraico, son familiares al alumno debido al estudio previo de la aritmética, por lo cual 
durante su enseñanza en la educación secundaria se presta poca atención al aprendizaje de dicha habilidad matemática (Kieran, 1992).

Esta investigación se sitúa en aquellos estudios que han abordado los procesos de generalización de patrones como medio en el desarrollo del pensamiento algebraico. De acuerdo con los trabajos que dan cuenta de las dificultades que presentan alumnos de nivel medio (14-16 años) al momento de obtener la regla general en el contexto de tareas sobre sucesiones de figuras (Becker \& Rivera, 2005; Barbosa, Vale \& Palhares, 2009) se mencionan: a) El predominio en el uso de estrategias aritméticas y recursivas, en detrimento de las de tipo visual o explícitas que permitan inducir el patrón de regularidad, b) La dificultad de expresar las relaciones generales, ya sea en representación verbal o simbólica, c) El manejo desprovisto de significado de una expresión simbólica, al margen del análisis contextual de las figuras de una sucesión.

En México la prueba Excale $e^{3}$ aplicada en el año 2008 expuso que 17\% de los estudiantes evaluados lograron establecer el patrón de una sucesión de figuras y usar esta expresión general para obtener el valor de cualquier término de la sucesión (Instituto Nacional de Evaluación de la Educación, INEE, 2009). Este dato es particularmente preocupante, pues revela la inhabilidad observada en los estudiantes, próximos a finalizar su educación media, para solucionar exitosamente tareas de generalización. Con ello se evidencia su poca habilidad para comprender nociones de variabilidad y el establecimiento de las relaciones generales.

Considerando la importancia que representa la propuesta de generalización de patrones en el contexto de la formación matemática escolar, particularmente el desarrollo del pensamiento algebraico, en esta investigación se propuso explorar, a partir de una primera fase de diagnóstico, cuáles fueron las estrategias de generalización usadas por los participantes en tareas de sucesión de figuras. Posterior a esta fase se planteó promover el uso de la estrategia visual como estrategia de inducción de la regla.

3 El Examen de la Calidad y Logro Escolar consiste en pruebas de gran escala aplicadas a muestras representativas de la población escolar. Son exámenes alineados al currículo escolar cuyo objetivo es contribuir al conocimiento del sistema escolar mexicano respecto a los aprendizajes escolares de los alumnos. Se trata de pruebas matriciales, criteriales y a gran escala, organizadas por el Instituto Nacional de Evaluación de la Educación (INEE) 


\section{MARCO CONCEPTUAL}

\section{Pensamiento algebraico}

En su trabajo doctoral Lins (1992) señaló que el pensamiento algebraico es visto como una habilidad para transitar del análisis del contexto a la estructura. Destreza que se aprecia cuando el estudiante logra "identificar propiedades generales que son instanciadas en situaciones particulares como relaciones entre los elementos" (Mason, Stephen \& Watson, 2009: 10). La posibilidad de identificar dichas relaciones permite al individuo establecer una "estructura" o patrón que ejemplifica la propiedad general. Además de lo anterior, Radford (2006) agrega como aspecto fundamental del pensamiento algebraico la capacidad de hacer uso de representaciones semióticas para externar este razonamiento de generalidad (simbolismos alfanuméricos, recursos verbales, gestos o ritmos). En los resultados que reporta Radford (2012) sobre la posibilidad que tienen los niños (de 8 a 13 años) de hacer objetiva una generalización de tipo algebraico, destaca el siguiente proceso:

a) Observar el aspecto común (semejanza y diferencia) entre los elementos que componen la sucesión;

b) Determinar un concepto -un género- que generalice lo común observado para todos los términos de la secuencia, y

c) Establecer una regla que exprese o integre a cualquier término de la secuencia.

\section{LA GENERALIZACIÓN COMO VÍA EN EL DESARROLLO DEL PENSAMIENTO ALGEBRAICO ESCOLAR}

La generalización de patrones es considerada una de las formas más importantes para introducir y desarrollar el pensamiento algebraico en las escuelas (National Council of Teachers of Mathematics, NCTM, 2000). El proceso de generalización permite que los estudiantes comprendan situaciones de variación consideradas fundamentales en el desarrollo del pensamiento algebraico y constituye una forma eficaz para introducir el estudio del álgebra en las escuelas (Mason, Graham \& Johnston-Wilder, 2012). De acuerdo con Walkowiak (2014: 57) "las experiencias sistemáticas con patrones, pueden construir una comprensión de la idea de 
función, la experiencia con números y sus propiedades, que representen una base para el trabajo posterior con símbolos y expresiones algebraicas". Por su parte, Rivera y Becker (2007) afirman que el propósito de la generalización en el contexto escolar es desarrollar la habilidad de expresar lo generalizable a partir del estudio analítico de casos particulares, de una forma significativa para el estudiante, como válida desde el punto de vista de la práctica institucional.

\section{Estrategias de generalización en sucesión de figuras}

En la Educación Matemática, las estrategias se definen como las formas de actuación o ejecución de tareas, dentro de una estructura que supone cualquier tipo de procedimiento que pueda efectuar, teniendo en cuenta las relaciones y los conceptos implicados. Gümer, Ersoy y Témiz (2013) sintetizan los modos de actuación de los sujetos ante la tarea de generalización en sucesiones de tipo figural y reportan las siguientes estrategias, presentadas en la Tabla 1.

Tabla 1. Estrategias de generalización de patrones.

\begin{tabular}{|c|c|}
\hline Estrategias & Propiedades \\
\hline Conteo & $\begin{array}{l}\text { Incluye el cálculo numérico de los términos a partir de la diferencia } \\
\text { constante entre cada uno de ellos. }\end{array}$ \\
\hline $\begin{array}{l}\text { Recursiva- } \\
\text { aditiva }\end{array}$ & $\begin{array}{l}\text { Se caracteriza por la obtención de términos a partir del uso del término } \\
\text { previo. }\end{array}$ \\
\hline $\begin{array}{l}\text { Multiplicar con } \\
\text { las diferencias }\end{array}$ & $\begin{array}{l}\text { En este caso los estudiantes, a partir de la diferencia constante entre los } \\
\text { términos, expresan el valor de cada término como la multiplicación del } \\
\text { término por la diferencia. }\end{array}$ \\
\hline Contextual & $\begin{array}{l}\text { Se construye una regla enfocada en el contexto, es decir, sobre la } \\
\text { información relacionada con la situación. }\end{array}$ \\
\hline Explícita & $\begin{array}{l}\text { Involucra la relación entre dos variables, a fin de determinar cualquier } \\
\text { término. Determina una función mediante el uso de la expresión general } \\
\text { que puede ser usada para encontrar } n \text { término de la sucesión. }\end{array}$ \\
\hline
\end{tabular}

Nota: Adapted de "7th $y 8^{\text {th }}$ Grade Students' Generalization Strategies of Patterns",

P. Guner, E. Ersoy \& T. Temiz, 2013, International Journal of Global Education, 2(4): 41. 
La diversidad de estrategias es resultado de razonamientos llevados a cabo por los estudiantes. Epistémicamente, se distinguen la segunda y tercera estrategias presentadas en la Tabla 1 por su naturaleza aritmética; en ellas el estudiante opera sobre algoritmos numéricos como medio para calcular los términos particulares de una sucesión. En contraste, la cuarta estrategia supone la lógica de un análisis visual de los elementos y sus relaciones con la figura, por esta razón se considera una estrategia de naturaleza visual y, a decir de Radford (2006), ejemplifica el tipo de generalización algebraica. La última estrategia consiste en la habilidad para construir una expresión algebraica que represente las relaciones funcionales y la posibilidad de obtener cualquier término de la sucesión.

Barbosa y Vale (2015) reconocen la existencia de factores que influyen en el modo de actuación de los alumnos en tareas de generalización:

a) El tipo de tarea con la cual se trabaja, que puede corresponder a sucesiones de primer orden (lineales, también llamadas transparentes) y de segundo orden (cuadráticas o no transparentes).

b) El tipo de preguntas que promueven el pensamiento relacional y reversible.

c) Solicitar generalizaciones, tanto cercanas como lejanas. Por generalización cercana se entiende obtener términos próximos a los observados; la generalización lejana implica inducir el término general que permite obtener los valores que no se pueden construir por medio del conteo.

Como propósito de enseñanza resulta entonces necesario que los estudiantes comprendan el potencial y limitaciones de cada uno de estos modos de actuación. No obstante, los resultados en investigaciones han reiterado la importancia de promover en las aulas la estrategia de visualización como el medio más eficaz y pertinente en el desarrollo de las habilidades necesarias en la inducción de patrones y su generalización y, con ello, el desarrollo del pensamiento algebraico (Arcavi, 2003; Radford, 2006; Barbosa \& Vale, 2015).

\section{El papel de la visualización en la generalización}

Arcavi (2003: 217) define la visualización como "una habilidad, proceso y producto de creación, interpretación, uso y reflexión sobre representaciones externas como dibujos, imágenes o diagramas en nuestras mentes con el propósito de 
representar y comunicar información". Para este autor la visualización ofrece un método de ver lo invisible, refiriendo con ello la posibilidad de percibir un mundo matemático abstracto solo visible a partir de la actividad humana.

Como habilidad, la visualización posibilita el análisis de una solución a un problema o la comprensión de un concepto u objeto matemático, y su aprendizaje (Ramírez, Ramírez, Flores \& Castro, 2013). En este sentido, la habilidad visual permite al alumno organizar y controlar datos a partir de la manipulación de objetos concretos, con la finalidad de establecer la solución formal a un problema (Rognes, 2010).

Como proceso, Presmeg (2006) afirma que la visualización implica transformar una representación externa en formas de representación interna. Según Duval (2006), para favorecer el aprendizaje matemático, desde la enseñanza han de promoverse actividades de conversión entre los distintos registros de representación; de tal forma, la visualización posibilita la conversión entre representaciones de un mismo objeto matemático.

Las representaciones externas son aquellos objetos que se manipulan en la actividad de visualización, en donde se realizan dos tipos de procesos (Bishop, 1989):

- Interpretación de información figurativa (IFI), que consiste en la comprensión de las representaciones visuales con el objetivo de extraer la información contenida, a partir de la manipulación de representaciones e imágenes. Se refiere al estímulo material.

- El procesamiento visual (VP), proceso de conversión de información abstracta o no figurativa en imágenes visuales, así como el proceso de transformación de unas imágenes visuales ya formadas en otras. Se trata de una capacidad de proceso y no se refiere a la forma del estímulo del material presentado. Su naturaleza es privada y personal.

En calidad de producto, Arcavi (2003) señala la visualización como un generador de representaciones internas. En suma, la visualización es un componente central en la actividad matemática (Rivera, 2011) que posibilita comprender objetos matemáticos complejos y abstractos (función) a partir del análisis de representaciones externas (figuras) con el fin de generar representaciones internas y cuyo resultado es la generalización de patrones, donde la representación semiótica es una regla general verbal o simbólica. 


\section{LA GENERALIZACIÓN DE SUCESIONES}

Sucesión de números naturales es un concepto cuya base se encuentra en la noción de conjunto ordenado con primer elemento y proceso infinito: para todo término de la sucesión hay un siguiente. Cuando se presentan varios términos de una sucesión y se pide continuar, la propuesta consiste en encontrar nuevos términos que mantengan relaciones con los ya conocidos (Rico, Castro \& Romero, 2000). Se denomina término a cada uno de los elementos o valores que constituyen el conjunto ordenado de la sucesión. En la literatura se escribe $a_{1}, a_{2}$, $a_{3}, \ldots a_{n}$, donde la expresión a representa el término de una sucesión y el subíndice indica el lugar al que está asociado dicho término. Por su parte, la expresión designa el término general de la sucesión. El reconocer las relaciones existentes entre los términos observados permite continuar la sucesión; por consiguiente, la caracterización general de una sucesión está representada mediante el término $a_{n}$.

Centrados en tareas de sucesiones de crecimiento lineal con representación de figuras, éstas se constituyen como una tarea rica en contexto para la tarea de generalización. Esta forma de representación se caracteriza por ser una secuencia de figuras que cambian de modo predecible a partir de una figura inicial, donde el objetivo es analizar, describir, ampliar el patrón y finalmente generalizar la relación de crecimiento (Walkowiak, 2014).

\section{METODOLOGÍA}

El estudio adoptó la metodología de Experimento de Enseñanza, método de investigación de naturaleza cualitativa cuyo objetivo permite analizar cómo se logra un aprendizaje en contexto de aula a partir del diseño de estrategias, conjeturas y herramientas de enseñanza (Molina, Castro, Molina \& Castro, 2011). Para Molina, Castro y Castro (2007) una de las características fundamentales de este método es el interés del investigador-docente por comprender de primera fuente los procesos de aprendizaje de los alumnos ante cierta habilidad o contenido, y tiene por tarea el proceso iterativo de diseñar, implementar, rediseñar y evaluar el Experimento.

En esta investigación se partió de una fase diagnóstica que permitió explorar el tipo de estrategias y las frecuencias con que fueron utilizadas en la obtención de la regla general. Posteriormente, los resultados generados 
sirvieron de insumo para planificar la fase de intervención didáctica, la cual consistió en cuatro sesiones y cuyo objetivo fue promover la adquisición y el uso de la estrategia visual como heurístico en la inducción del patrón de crecimiento. En este documento se reporta el tercer episodio de intervención, dado que fue donde se logró evidenciar la eficacia de la estrategia visual en la tarea de inducir la regla.

\section{PARTICIPANTES}

Intervinieron 28 alumnos en la fase diagnóstico y 30 en la intervención. Las edades estuvieron comprendidas entre los 14 y 16 años. Fueron 17 mujeres y 13 hombres. 18 estudiantes egresaron de la modalidad de Secundaria General y el resto proviene de escuelas Telesecundarias. Se consideró como único criterio de selección el que fueran estudiantes de recién egreso de la educación secundaria, pues fue de interés identificar las estrategias de generalización logradas en la formación matemática de este nivel. Con base en la revisión del plan de estudios, se infiere que los participantes tenían experiencia previa en el trabajo sobre sucesión de figuras. El Experimento se llevó a cabo durante los meses de septiembre a octubre del año 2016.

\section{EL PAPEL DEL INVESTIGADOR-DOCENTE}

A continuación se detallan las actividades del investigador-docente en el Experimento:

- Antes de la intervención definió: a) Objetivos de aprendizaje esperados a partir de la revisión teórica del concepto pensamiento algebraico, b) El tipo de tarea (sucesión) presentada a los alumnos, c) Los materiales de trabajo. Además, detalló las estrategias del acopio de datos.

- Al inicio de cada sesión explicó a los estudiantes la naturaleza del trabajo y el tiempo destinado para la resolución de la tarea propuesta.

- Durante el desarrollo de la sesión orientó el trabajo de los participantes hacia el logro de los objetivos propuestos. Las decisiones de enseñanza se encaminaron en promover las habilidades visuales como estrategia de generalización. Atendió las dudas que surgieron en el avance de las actividades. 
- En el análisis de los resultados, el investigador identificó los cambios observados en la habilidad de establecer la expresión general mediante la estrategia de visualización. Con base en esta interpretación se identificaron aquellas acciones específicas que contribuyeron a promover la habilidad de generalización en sucesión de figuras y del pensamiento algebraico.

\section{TÉCNICAS DE OBTENCIÓN DE DATOS}

Se obtuvo registro de las hojas de trabajo de cada una de las ocho tareas implementadas y que los estudiantes resolvieron en la fase diagnóstica, las cuatro sesiones de intervención y la sesión de entrevistas individuales. La información recogida, a partir de las hojas de trabajo, permitió identificar el tipo de estrategias utilizadas por los alumnos en la tarea de construir la regla general y valorar la modificación del tipo de respuesta al incorporar la estrategia de visualización; además, permitió al investigador-docente evaluar las frecuencias de respuestas correctas en el logro de la tarea en la sesión de diagnóstico y contrastarlas con las frecuencias de éxito en la tercera sesión que aquí se reporta.

Otro de los medios de acopio de información consistió en la grabación en video de las sesiones de trabajo. Con estos registros fue posible identificar el tipo de argumentos que externaron los estudiantes respecto al tipo de estrategia de generalización. Asimismo, se obtuvo evidencia del trabajo de campo mediante la observación directa en clase. Por último, el investigador-docente registró en notas de campo las estrategias y dificultades observadas por los alumnos en el momento de la resolución de las tareas. Estas evidencias fueron entrelazadas con el registro de las transcripciones de los videos.

\section{TAREAS}

Enseguida se muestran las dos tareas reportadas en este trabajo. La primera corresponde a una de las presentadas en la fase de diagnóstico (Figura 1). Esta sucesión mostró al alumno los tres primeros términos y se requirió obtener $a_{5}$, $a_{10}, a_{100}, a_{n}$. Cada término consistió en dos filas de puntos con la expresión $2 n+3$ como expresión algebraica asociada a la regla. 
Figura 1. Sucesión de puntos. Fase diagnóstico.

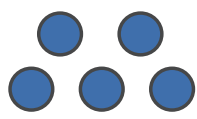

$a_{1}$

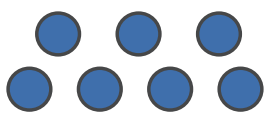

$a_{2}$

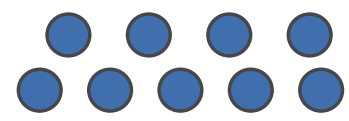

$a_{3}$

La segunda tarea (Figura 2) mostró una sucesión con términos ocultos y se pidió derivar los términos $a_{7}, a_{10}, a_{100}, a_{n}$. Cada término se estructura de una fila de líneas inferiores, superiores e intermedios. Se esperó que el alumno construyera la expresión como patrón de crecimiento.

Figura 2. Sucesión con término ocultos. Fase de intervención.

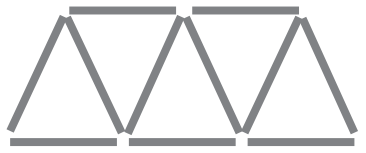

$a_{3}$

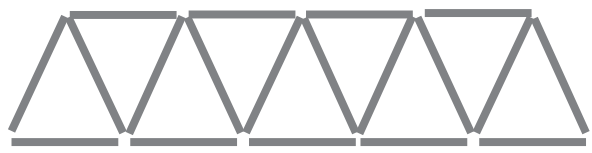

$a_{5}$

\section{CATEGORIZACIÓN DE LOS DATOS}

Se presenta en dos momentos. La primera es de tipo apriorístico, es decir, construida antes del acopio de datos y generada a partir de la revisión bibliográfica antecedente.

$>$ Categorías previas:

- Diferencia entre los términos (Df): consiste en obtener el valor de la diferencia aritmética entre dos términos de una sucesión;

- Conteo (Co): a partir de la estrategia precedente, el estudiante aplica una suma de la diferencia entre los términos para encontrar el término solicitado;

- Visual (Vi): el alumno establece una representación analítica y relaciona los elementos de la figura para obtener la estructura general del término de la sucesión;

- Multiplicativo con ajuste (MA): se centra en construir una función a partir del valor de la diferencia constante entre dos términos, multiplicando este valor por el número del lugar del término, para ajustarlo finalmente al valor numérico del término. 
La segunda corresponde a la categorización emergente, resultado del proceso de análisis de intervención didáctica.

> Categorías emergentes:

1. Agrupada en Relación a la Figura (ARF). Se caracteriza porque el alumno, mediante la estrategia visual, logró identificar un número de triángulos y de líneas superiores. El patrón en representación de la regla verbal fue: por cada término estuvo asociado igual número de triángulos y, para completar la estructura, por cada figura se asoció un número de líneas superiores en relación a menos uno con el número de figura. La expresión general resultante estaría dada por el valor $3_{n}$ que corresponde al número de triángulos de la figura, más la expresión $n-1$ que se relaciona con el número de líneas superiores. La reducción da como resultado la expresión $a_{n}=3_{n}+(n-1)=4_{n}-1$ que representa la estructura de la sucesión.

2. Fragmentada en Relación a la Base (FRB). Se fragmentó la figura e identificaron tres elementos (líneas de base, internas, superiores). La regla verbal y la relación entre estos fue establecida de la siguiente forma: las líneas de base se corresponden con el término y el símbolo $n$; las líneas superiores tienen una relación con el número de líneas de base menos uno, las asocia la expresión $n-1$; las líneas internas guardan una relación del doble con respecto al número de líneas de base, $2 n$. Al reducir las expresiones anteriores se obtiene la regla $a_{n}=n+(n-1)+2 n=4_{n}-1$.

3. Incompleta Agrupada en Relación a la Figura (IARF). Se caracteriza porque el alumno logró identificar el número de triángulos mencionado en ARF, pero no logra establecer la relación del elemento de las líneas superiores en la estructura general del término. La expresión que se estableció fue $a_{n}=3 n$.

4. Incompleta Fragmentada en Relación a la Base (IFRB). El estudiante estableció los elementos de líneas de base e internas, pero al momento de expresar la estructura general no articuló el elemento de líneas superiores.

5. Traducción de la Regla Aritmética (TRA). El alumno, tras haber obtenido la regla general mediante la estrategia de DfMA, hizo una traducción de la expresión simbólica al modo verbal.

6. NR. No Respondió. 


\section{DESARROLLO DE LAS SESIONES}

En la sesión de diagnóstico participaron 28 estudiantes y la clase tuvo una duración de 40 minutos. Se planteó como objetivo de investigación identificar las estrategias de generalización de patrones. La tarea de diagnóstico que se reporta en este artículo consistió en una sucesión de tres términos consecutivos, cada figura se conformó por dos filas de puntos horizontales (ver Figura 1). La resolución de las tareas fue de forma individual.

En la fase de intervención participaron 30 alumnos y constó de cuatro sesiones. El episodio de enseñanza que aquí se reporta corresponde a la tercera sesión, pues fue donde se consiguió que un mayor número de participantes lograran inducir correctamente el patrón de regularidad. El antecedente de esta sesión fue la preeminencia de la estrategia DfMA al obtener la regla general.

Para esta tercera sesión y con los antecedentes de la primacía de la estrategia de generalización DfMA, decidimos continuar con la sucesión de términos ocultos, pues se conjeturó que esta forma de presentar la sucesión limitaría el uso de estrategias recursivas o aritméticas (Samson, 2012). Además de la incorporación de la variable tarea, se planteó valorar el impacto de la manipulación de objetos concretos (palillos de madera) como estrategia visual encaminada a inducir la regla. De este proceso el estudiante daría cuenta ya no de casos concretos (contexto), sino que sería capaz de describir y extender la estructura general de la sucesión. En consecuencia, una vez inducido un patrón de regularidad, el alumno tendría la posibilidad de expresar el término general. La sesión que se reporta tuvo una duración de 50 minutos.

\section{RESULTADOS}

\section{ACERCA DE LA SESIÓN DIAGNÓSTICO}

La Tabla 2 muestra las frecuencias de respuesta correcta por término solicitado en la tarea de diagnóstico, con relación al tipo de estrategia usada y el modo de representación de la regla. 
Tabla 2. Respuestas correctas, estrategias y representación del término general.

\begin{tabular}{ccrcccc}
\hline & & \multicolumn{3}{c}{ Estrategias } & \multicolumn{2}{c}{ Representación de $\mathrm{a}_{\mathrm{n}}$} \\
\cline { 3 - 7 } Término & Frecuencia de RC & Co & Vi & DfMA & Si & Ve \\
\hline $\mathrm{a}_{5}$ & 22 & 12 & 2 & 8 & & \\
$\mathrm{a}_{10}$ & 17 & 8 & 0 & 9 & & \\
$\mathrm{a}_{100}$ & 12 & 0 & 0 & 12 & & \\
$\mathrm{a}_{\mathrm{n}}$ & 10 & 0 & 0 & 10 & 10 & 0 \\
\hline
\end{tabular}

Nota: $\mathrm{Co}=$ conteo; $\mathrm{Vi}$ = visual; $\mathrm{Df}=$ diferencia; $\mathrm{MA}=$ multiplicativo con ajuste; $\mathrm{Si}=$ simbólico; $\mathrm{Ve}=$ verbal. Fuente: Elaboración propia.

En la segunda columna se observa que las frecuencias de RC disminuyen conforme se incrementa la posición del término solicitado. En la actividad de obtener la expresión de $a_{n} 35 \%$ de los estudiantes lograron resolver correctamente la actividad. La estrategia utilizada en la totalidad de estos casos de RC fue la de diferencias con factor multiplicativo y ajuste. Se destaca también la preponderancia de la regla simbólica como la forma de representar el término $a_{n}$.

Otro aspecto identificado es el predominio de la estrategia de conteo para obtener los valores cercanos $a_{5}$ y $a_{10}$. Esta estrategia de recursividad permitió a los participantes obtener términos cercanos al sumar al término anterior el valor de las diferencias entre dos términos consecutivos, sin embargo, dicha estrategia dejó de ser efectiva al momento de obtener el valor de los términos $a_{100}$ y $a_{n}$. Se observó que la estrategia de sumar dos al término anterior fue depuesta, pues representó continuar la operación de sumar de 2 en 2 hasta llegar al valor del término 100. Por último, la estrategia de visualización fue empleada por dos alumnos para obtener el valor de $a_{5}$ y dejó de ser utilizada en el resto del trayecto de la tarea de diagnóstico.

Estos hallazgos sugieren que los alumnos con RC, para el término general, iniciaron con la estrategia de conteo, transformando la representación de figuras en valores numéricos, y sobre ellos se operó de forma aritmética para obtener la expresión simbólica. No obstante, en este modo de generalización aritmética se limitó la posibilidad de establecer generalizaciones algebraicas a partir del análisis y establecimiento de relaciones generales en las figuras o términos de la sucesión. 


\section{ACERCA DE LA INTERVENCIÓN DIDÁCTICA}

A continuación se reporta el trabajo recogido en uno de los grupos participantes donde se observó un proceso de inducción mediante la manipulación de material concreto. El grupo que se reporta estuvo conformado por Genaro, Rocío, Esteban y José. En este acercamiento Genaro intentó establecer una estructura con base en el análisis del término $a_{3}$. Basado en el conteo de líneas inferiores más las superiores, encontró relación con el número de triángulos y expuso:

Genaro: el número de triángulos es el mismo número que el de las líneas de arriba más las de abajo.

El alumno comprendió que en el término 3 de la sucesión había cinco triángulos, los cuales estaban en relación con la suma de las líneas de base, tanto inferiores como superiores de los triángulos. Es decir, el patrón que el alumno identificó se puede representar con la expresión:

$$
a_{n}=n+(n-1) \text { : donde } n \text { correspondía al número de triángulos }
$$

En la Figura 3 se observa el proceso de abstracción de la regla que el alumno construyó.

Figura 3. La señalización como recurso de objetivación del patrón.

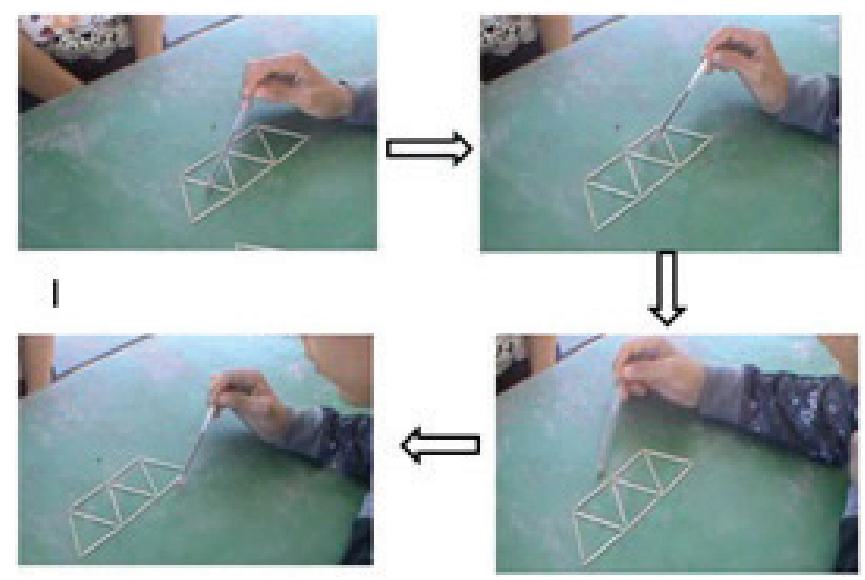


Al iniciar, el alumno formuló un conteo de lo que consideró la estructura de los triángulos que conforman la figura. En el recuadro superior izquierdo de la Figura 3 se aprecia que ubicó correctamente un triángulo, sin embargo, en el recuadro superior derecho señaló lo que consideramos una abstracción incorrecta, pues duplica el número de triángulos, lo cual deriva en una formulación incorrecta del patrón. En los recuadros inferiores el estudiante realizó un conteo de las líneas superiores e inferiores y con ello, como se presentará en el siguiente párrafo, logró inducir las relaciones entre las subestructuras que conforman la figura.

Una vez comprendida la inducción, el investigador-docente señaló al alumno que conocer el número de triángulos, bajo esta abstracción, no permitiría obtener el número de líneas correspondientes al término $a_{n}$ de la sucesión, pues se cometía el error de duplicar el número de líneas que forman la estructura en $\mathrm{V}$ del triángulo. Esteban, integrante de este equipo de trabajo, corrige y comenta que la tarea consiste en encontrar el número de líneas que componen la figura, no el número de triángulos. Esta observación fue importante porque permitió al equipo inducir nuevas relaciones entre las líneas que conforman la figura, sin necesidad de estructurar las líneas en triángulos. Con esta nueva forma de fragmentar la figura, los alumnos identificaron tres elementos: líneas de base, superiores e internas. Con base en esta abstracción Genaro continuó:

Genaro: encontré que el número de líneas que están en medio (internas) siempre es una menos que el total de los que están abajo y arriba como aquí (señala con su dedo índice el término $a_{5}$ ) son: 1, 2, 3, 4, 5 (cuenta las líneas inferiores), 6, 7, 8, 9 (sumando las líneas de base y superiores del término $a_{5}$ )... y acá son $1,2,3,4,5$, 6, 7, 8, 9, 10; (al hacer el conteo de las líneas internas, corrige) digo uno más. Aquí también (el alumno valida y amplía su inducción a los demás casos observados) 1 , 2, 3, 4, 5 (suma las líneas inferiores y superiores del término $a_{3}$ ). 1, 2, 3, 4, 5, 6 (cuenta de forma rítmica los palillos de en medio o zigzag).

Según lo observado, la visualización permitió al estudiante inducir el patrón a partir de la manipulación, fragmentación y análisis de la figura en elementos articulados que conforman el término: las líneas de en medio siempre fueron una más que la suma de las líneas inferiores y superiores; que el número de la figura siempre fue igual a las líneas de base. Este modo de actuación visual partió del análisis de los elementos que conforman cada uno de los términos particulares, para posteriormente extender el dominio de las relaciones inducidas a un contexto general o de estructura. 
La Figura 4 evidencia la forma en que Genaro planteó la descomposición de la figura en los elementos que la constituyen, y estableció las relaciones numéricas a partir del orden numérico y espacial que disponen dichos elementos en el contexto del término analizado. Es importante destacar el papel que desempeñaron los recursos de objetivación semiótica de la regla. En este caso, tales recursos fueron tanto la señalización indixecal de los elementos como el ritmo empleado en la articulación de las relaciones (Radford, 2006, 2010, 2012).

Figura 4. La señalización como recurso de objetivación de la regla general.

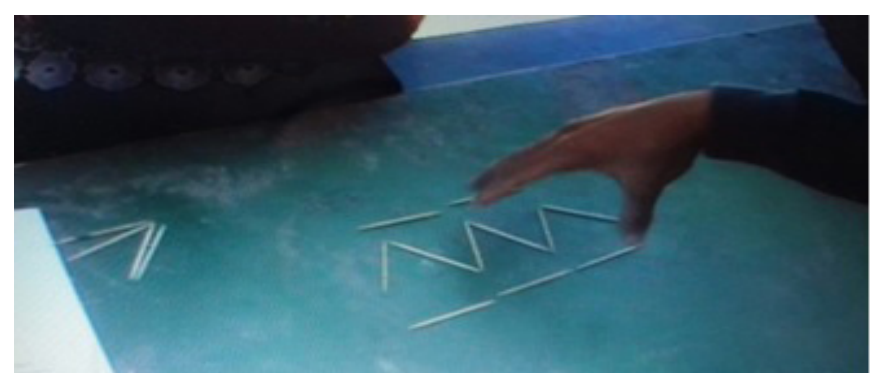

Fuente: Elaboración propia.

Enseguida se reporta lo observado por el grupo total de estudiantes en cuanto a la habilidad de inducir la regla mediante la manipulación de materiales concretos. La Tabla 3 expone la estrategia de generalización en la tercera sesión.

Tabla 3. Estrategia de generalización en sucesión con término oculto.

\begin{tabular}{cccc} 
& \multicolumn{3}{c}{ Estrategia de generalización } \\
\cline { 2 - 4 } Término & Vi & DfMA & NR \\
\hline$a_{n}$ & 22 & 2 & 6 \\
\hline
\end{tabular}

Nota: $\mathrm{Vi}=$ Visual; DfMA = Diferencia Multiplicativa con Ajuste; $\mathrm{NR}=$ No Respondió.

Fuente: Elaboración propia. 
Se observa que el uso de materiales concretos favoreció que 73\% de los participantes empleara la estrategia visual como recurso para establecer las relaciones estructurales en las figuras e inducir el patrón de crecimiento. Dos casos continuaron con el razonamiento aritmético para el establecer el valor de la regla y 6 estudiantes no respondieron a la tarea, lo cual supuso desinterés en los alumnos por explorar este tipo de estrategia visual.

En el contexto de la generalización aritmética DfMA, los estudiantes encontraron que, si bien no eran consecutivos, entre los términos $a_{3}$ y $a_{5}$ existía una diferencia de ocho líneas, en consecuencia, el valor de la diferencia entre dos términos consecutivos sería de 4 . Este valor fue tomado como factor multiplicativo de la variable independiente (lugar del término o número de figura) y su resultado fue la expresión $4_{n}$. El producto de la multiplicación de la expresión $4_{n}$ se ajustó al número de líneas de cada figura; por ejemplo, para la figura tres se multiplicó 4 x $3=12$, entonces el ajuste consistió en restar 1 , dando como solución la expresión $a_{n}=4_{n}-1$.

Como resultado de la estrategia visual se establecieron dos tipos de abstracciones. La Figura 4 muestra las categorías emergentes que representan los diferentes razonamientos, producto de la intervención didáctica, y las frecuencias con las que el total de estudiantes establecieron total o parcialmente el patrón.

Tabla 4. Abstracción visual del patrón de crecimiento en sucesión con término oculto.

\begin{tabular}{cccccc}
\hline & \multicolumn{3}{c}{ Abstracción visual del patrón en sucesión con término oculto } \\
\cline { 2 - 6 } Término & \multicolumn{2}{c}{ Patrón completo } & \multicolumn{2}{c}{ Patrón incompleto } \\
\hline$a_{n}$ & ARF & FRB & IARF & IFRB \\
\hline
\end{tabular}

Nota: $\mathrm{ARF}=$ Agrupada en Relación a la Figura; FRB = Fragmentada en Relación a la Base;

IARF = Incompleta Agrupada en Relación a la Figura; IFRB = Incompleta Fragmentada en Relación a la Base; TRA = Traducción de la Regla Aritmética; NR = No Respondió.

Fuente: Elaboración propia.

De los 15 estudiantes que lograron construir la regla general, 80\% recurrió a la fragmentación de la figura en líneas (FRB). Esta forma de abstraer las relaciones sugiere que el desintegrar la figura en el mayor número de elementos permitió al alumno establecer de forma efectiva las relaciones numéricas espaciales entre los 
elementos (líneas de base, intermedias y superiores). El 20\% restante estableció una inducción a partir de observar una subestructura (triángulos) en la figura (ARF). Si bien esta última abstracción fue la sugerida por parte del investigador-docente, el patrón basado en la agrupación de triángulos generó mayor confusión al tratar de comprender las relaciones entre los elementos en la figura, y produjo desconcierto al comprender si lo que se buscaba era el número de triángulos o de líneas. Este hallazgo respecto a la posibilidad de establecer relaciones estructurales de la figura, como aspecto indispensable para inducir un patrón, es similar al observado en el estudio de Callejo, García-Reche y Fernández (2016) y Cañadas, Castro y Castro (2008), autores que afirman que la generalización depende tanto de la detección de un patrón como de identificar un patrón adecuado.

Siete casos solo lograron establecer una regla incompleta. En estas construcciones parciales se observó que los estudiantes no consideraron las líneas superiores como elemento formativo de la estructura. Este hallazgo sugiere la necesidad de mayor familiaridad por parte del estudiante en tareas de construcción de patrones, mediante la estrategia de visualización. Por consiguiente, se infiere que la preminencia de las generalizaciones aritméticas, trabajadas en los niveles de educación que preceden al bachillerato, han soslayado las habilidades visuales de analizar y establecer relaciones en las figuras en una sucesión.

Respecto a las formas de representar la regla de crecimiento, se observó que los alumnos que establecieron una generalización visual recurrieron tanto a la verbalización como a la representación simbólica, como medio para expresar el patrón. La Tabla 5 muestra las frecuencias según el tipo de representación, se destaca el hecho de que la mayoría mostró en su hoja de respuesta los dos tipos de representación, simbólica y verbal.

Tabla 5. Representación de la regla según estrategia de generalización.

Estrategia de generalización

\begin{tabular}{ccccc}
\hline & Visual & & \multicolumn{2}{c}{ Aritmético } \\
$\mathrm{Si}$ & $\mathrm{Ve}$ & $\mathrm{Si}$ & $\mathrm{Ve}$ \\
\hline 18 & 22 & 2 & 2 \\
\hline
\end{tabular}

Nota: $\mathrm{Si}=$ Simbólico; $\mathrm{Ve}=$ Verbal.

Fuente: Elaboración propia. 
Al contrastar las Tablas 2 y 5 se reconoce que la estrategia visual permitió transitar de un uso predominante de la representación simbólica al establecimiento de las relaciones de forma verbal, favoreciendo con ello la capacidad de los alumnos para expresar y argumentar de forma verbal la estructura que se ha inducido. Por otra parte, dos estudiantes realizaron una traducción de la regla simbólica generada a partir de la estrategia DfMA a la representación verbal, pero sin atender a la naturaleza visual de la figura.

Por último, al comparar las Tablas 2 y 3 observamos que el uso de material concreto, al igual que el trabajo con sucesiones con términos ocultos, favoreció que los alumnos recurrieran a la estrategia visual como medio de generalización. Se logró que $73 \%$ de los participantes en la fase de intervención recurrieran a la estrategia visual como modo de generalización; de ellos, 68\% estableció un patrón válido de crecimiento y pudo representarlo mediante una regla verbal. Cualitativamente, la estrategia visual permitió que los estudiantes centraran su razonamiento en establecer nociones generales de variabilidad a partir del análisis de los términos en sucesión, en tanto que la actividad sobre patrones de figuras no se limitó a efectuar solamente cálculos aritméticos encaminados a la obtención de un valor numérico como respuesta.

\section{CONCLUSIONES}

En un primer momento se reconoce la preponderancia que significaron las estrategias aritméticas en la construcción del término general, lo cual fue evidente en la fase de diagnóstico. Este hallazgo puede deberse a la familiaridad de los participantes para trabajar con operaciones aritméticas en contextos cotidianos (ver Tabla 2). Como medio para expresar la regla, se identificó además que la totalidad de los alumnos establecieron la regla simbólica del patrón, probable resultado de un entrenamiento en el uso del lenguaje alfanumérico, surgido de la formación matemática en el nivel de educación secundaria; no obstante esta habilidad en el manejo de lo simbólico, las expresiones sirvieron como fórmula para generar una respuesta numérica al problema de obtener el número de puntos para un término particular de la sucesión, y no como medio para expresar la variabilidad en el contexto de la sucesión de figuras.

Respecto al establecimiento de las generalizaciones algebraicas, los hallazgos en la sesión de intervención permiten evidenciar el desarrollo en la habilidad de inducir relaciones generales a partir del análisis visual de cada uno de los 
elementos que conforman un término de la sucesión. En este sentido, la visualización permitió a los alumnos establecer relaciones numérico-espaciales (ver Figura 4), extendiendo el dominio de este razonamiento al término o patrón general de la sucesión (Del Grande, 1990). El proceso de visualización partió de la manipulación de objetos concretos, actividad que permitió fragmentar la figura e identificar cada uno de los elementos que la forman; posteriormente los alumnos hicieron el conteo de cada uno de los elementos y establecieron las relaciones en cada término para, finalmente, inducir la validez del patrón a los demás términos de la sucesión.

En términos de eficacia, se mostró que fue mayor el número de estudiantes que lograron establecer una generalización algebraica mediante el uso de la estrategia visual descrita, en comparación con 35\% de los alumnos que recurrieron a la generalización mediante razonamientos aritméticos (ver Tabla 3). Con la implementación de la estrategia visual se consiguió que 50\% de los participantes establecieran un patrón de generalidad y lograran verbalizar la regla de crecimiento.

Al contrastar los medios de expresar el patrón, entre la fase de diagnóstico y de intervención, se observó que 73\% del total de estudiantes expresó la inducción de la regla mediante la verbalización de las relaciones producto de la estrategia visual. Este hallazgo permite valorar esta forma verbal de introducir y representar ideas matemáticas relacionadas con fenómenos de variabilidad. Junto con este hallazgo se destaca también $80 \%$ de los alumnos que, junto con la verbalización, emplearon las expresiones simbólicas para denotar la regla de una sucesión.

No obstante, observamos también que la inducción del patrón general resultó ser un proceso complejo en el momento de articular y expresar la regla, ya sea en su modo de representación verbal o simbólica. En los siete casos en donde se estableció una parcialidad del patrón, se observó la necesidad de mayor familiaridad con este tipo de tareas, que promuevan y familiaricen en el alumno el poder establecer relaciones generales que atiendan la totalidad de la estructura.

Consideramos dos implicaciones didácticas de esta experiencia. La primera relacionada con la importancia de promover en el aula, desde los niveles de educación básica, estrategias de visualización que fomenten en el estudiante la capacidad de inducir, validar y expresar relaciones generales mediante la descomposición analítica de las figuras, más que abordar este tipo de tareas de generalización desde una estrategia o razonamiento aritmético en donde el 
alumno se limita a construir una expresión derivada de un enfoque donde la atención está en la obtención de un valor numérico (Blanton \& Kaput, 2011). La segunda implicación es la solvencia por parte del docente en el manejo de los distintos patrones que puedan surgir en el proceso de inducción. Esta implicación deriva en hacer investigaciones que exploren el tipo de enseñanza que promueva la propuesta de generalizaciones algebraicas en los contenidos de sucesiones de figuras. El reconocimiento de la diversidad de inducciones permitirá que el profesor identifique los diferentes razonamientos llevados a cabo por los alumnos y atender las dificultades que pudieran surgir ante este tipo de tareas, buscando fortalecer desde la enseñanza la habilidad de inducir patrones para con ello promover el desarrollo del pensamiento algebraico mediante los procesos de generalización.

\section{REFERENCIAS}

Arcavi, A. (2003). The Role of Visual Representations in the Learning of Mathematics. Educational Studies of Mathematics, 52(3), 215-241.

Barbosa, A., Vale, I. \& Palhares, P. (2009). Exploring Generalization with Visual Patterns: Tasks Developed with Pre-algebra Students. Padrōes: Múltiplas Perspectivas e Contextos em Educação Matemática, 137-149.

Barbosa, A. \& Vale, I. (2015). Visualization in Pattern Generalization: Potential and ChaIlenges. Journal of the European Teacher Education Network, 10, 57-70. Recuperado de http://www.jeten-online.org/index.php/jeten/article/view/67.

Becker, J. \& Rivera, F. (2005). Generalization Strategies of Beginning High School Algebra Students. En H. Chick \& J. Vincent (eds.), Proceedings of the 29th Conference of the International Group for the Psychology of Mathematics Education, Vol. 4, pp. 121-128. Melbourne: University of Melbourne.

Bishop, A. (1989). Matemática y Educación: Retos y cambios desde una perspectiva internacional. Ed. Graó. Barcelona.

Blanton, M. \& Kaput, J. (2011). Functional Thinking as a Route into Algebra in the Elementary Grades. International Reviews on Mathematical Education, 37(1), 34-42. DOI: 10.1007/BF02655895.

Callejo, M., García-Reche, Á. \& Fernández, C. (2016). Pensamiento algebraico de estudiantes de educación primaria (6-12 años) en problemas de generalización de patrones lineales. Avance de Investigación en Educación Matemática, 10, 5-25. 
Cañadas, M., Castro, E. \& Castro, E. (2008). Patrones, generalización y estrategias inductivas de estudiantes de $3^{\circ}$ y $4^{\circ}$ de Educación Secundaria Obligatoria en el problema de las baldosas. PNA, Revista de Investigación en Didáctica de la Matemática, 2(3), 137-151.

Del Grande. J. (1990). Spatial Sense. Arithmetic Teacher, 37(6), 14-20.

Duval, R. (2006). Un tema crucial en la educación matemática: La habilidad para cambiar el registro de representación. La Gaceta de la RSME, 9(1), 143-168.

Gümer, P., Ersoy, E. \& Témiz, T. (2013). 7th and 8th Grade Students' Generalization Strategies Patterns. International Journal of Global Education-2013, (2)4, 38-45.

Instituto Nacional de Evaluación de la Educación. (2009). El aprendizaje de las matemáticas. El aprendizaje en tercero de secundaria en México. Informe sobre los resultados del Excale 09, aplicación 2008 (pp. 91-113), México: INEE.

Kieran, C. (1992). The Learning and Teaching of School Algebra. En D. A. Grouws (ed.), The Handbook of Research on Mathematics Teaching and Learning, (pp. 390-419). New York: Macmillan.

Lins, R. (1992). A Framework for Understanding what Algebraic Thinking Is (Tesis doctoral). Universidad de Nottingham, UK.

Mason, J., Stephens, M. \& Watson, A. (2009). Appreciating Mathematical Structure for All. Mathematics Education Research Journal. 21(2), 10-32.

Mason, J., Graham, A. \& Johnston-Wilder, S. (2012). Developing Thinking in Algebra. Londres, Inglaterra: The Open University.

Molina, M. (2009). Una propuesta de cambio curricular: integración del pensamiento algebraico en educación primaria. PNA, Revista de Investigación en Didáctica de la Matemática, 3 (3), 135-156.

Molina, M., Castro, E. \& Castro, E. (2007). Teaching Experiments within Design Research. The International Journal of Interdisciplinary Social Sciences, 2(4), 435-440.

Molina, M., Castro, E., Molina, J. \& Castro, E. (2011). Un acercamiento a la investigación de diseño a través de los experimentos de enseñanza. Enseñanza de las Ciencias: Revista de Investigación y Experiencias Didácticas, 29(1), 75-88.

National Council of Teachers of Mathematics. (2000). Principles and Standards for School Mathematics. Recuperado de https://www.nctm.org/uploadedFiles/Standards_and_ Positions/PSSM_ExecutiveSummary.pdf.

Presmeg, N. (2006). Research on Visualization in Learning and Teaching Mathematics. Handbook of Research on the Psychology of Mathematics Education: Past, Present and Future. Sense Publishers, Rotterdam, 205-235.

Radford, L. (2006). Algebraic Thinking and the Generalization of Patterns: A Semiotic Perspective. En S. Alatorre et al. (eds.), Proceedings of the 28th Anual Meeting of the 
North American Chapter of the International Group for the Psychology of Mathematics Education, North America Chapter, Vol. 1, pp. 2-21. Mérida: Universidad Pedagógica Nacional.

Radford, L. (2010). Layers of Generality and Types of Generalization in Pattern Activities. PNA, Revista de Investigación en Didáctica de la Matemática, 4(2), 37-62.

Radford, L. (2012). On the Development of Early Algebraic Thinking. PNA, Revista de Investigación en Didáctica de la Matemática, 6(4), 117-133.

Ramírez, R., Ramírez, I., Flores, P. \& Castro, E. (2013). "Análisis de las capacidades de visualización espacial e intelectual en los alumnos con talento matemático". Revista Mexicana de Psicología, 30(1), 267-299.

Rico, L., Castro, E. \& Romero, I. (2000). Sistemas de representación y aprendizajes de estructuras numéricas. Granada, España: Universidad de Granada.

Rivera, F. \& Becker, J. (2007). Abduction-Induction (Generalization) Processes of Elementary Majors on Figural Patterns in Algebra. The Journal of Mathematical Behavior, 26(2), 140-155. Recuperado de http://dx.doi.org/10.1016/j.jmathb.2007.05.001.

Rivera, F. (2011). Visual Roots of Mathematical Cognitive Activity. En A. Bishop (ed.), Toward a Visually-Oriented School Mathematics Curriculum. Springer. Recuperado de http://doi.org/10.1007/978-94-007-0014-7.

Rognes, J. (2010). Mathematical Visualization. Journal of Mathematics Education at Teachers College. Technology Issue, Fall-Winter 2011, Vol. 2.

Samson, D. (2012). Encouraging Meaningful Engagement with Pictorial Patterning Tasks. Australian Mathematics Teacher, 68(2), 4-10.

Vergel, R. (2015). Generalización de patrones y formas de pensamiento algebraico temprano. PNA, Revista de Investigación en Didáctica de la Matemática, 9(3), 193-215.

Walkowiak, T. (2014). Elementary and Middle School Students' Analyses of Pictorial Growth Patterns. The Journal of Mathematical Behavior, 33, 56-71. Recuperado de https:// doi.org/10.1016/j.jmathb.2013.09.004. 